\title{
Effect of Direct Current on Solid-Liquid Interfacial Tension and Wetting Behavior of Ga-In-Sn Alloy Melt on Cu Substrate
}

\author{
Limin Zhang $\mathbb{D}^{\mathbb{D}},{ }^{1}$ Ning Li, ${ }^{1}$ Hui Xing, ${ }^{1}$ Rong Zhang $\mathbb{D}^{1},{ }^{1}$ and Kaikai Song ${ }^{2}$ \\ ${ }^{1}$ Shaanxi Key Laboratory of Condensed Matter Structures and Properties and Key Laboratory of Space Applied Physics and Chemistry, \\ Ministry of Education, School of Science, Northwestern Polytechnical University, 127 West Youyi Road, Xian, Shaanxi 710072, China \\ ${ }^{2}$ School of Mechanical, Electrical \& Information Engineering, Shandong University at Weihai, 180 Wenhua Xilu, \\ Weihai, Shandong 264209, China
}

Correspondence should be addressed to Rong Zhang; xbwl01@mail.nwpu.edu.cn

Received 22 November 2017; Accepted 11 January 2018; Published 1 March 2018

Academic Editor: Liyuan Zhang

Copyright (C) 2018 Limin Zhang et al. This is an open access article distributed under the Creative Commons Attribution License, which permits unrestricted use, distribution, and reproduction in any medium, provided the original work is properly cited.

\begin{abstract}
The effect of direct current (DC) on the wetting behavior of $\mathrm{Cu}$ substrate by liquid Ga-25In-13Sn alloy at room temperature is investigated using a sessile drop method. It is found that there is a critical value for current intensity, below which the decrease of contact angle with increasing current intensity is approximately linear and above which contact angle tends to a stable value from drop shape. Current polarity is a negligible factor in the observed trend. Additionally, the observed change in contact angles is translated into the corresponding change in solid-liquid interfacial tension using the equation of state for liquid interfacial tensions. The solid-liquid interfacial tension decreases under DC. DC-induced promotion of solute diffusion coefficient is likely to play an important role in determining the wettability and solid-liquid interfacial tension under DC.
\end{abstract}

\section{Introduction}

During the solidification process of materials, the application of electric current including direct current, alternating current $(\mathrm{AC})$, and electric current pulse (ECP) has developed into a promising technique to modify the solidifying structure in the past decades due to their high efficiency and cleanliness [1-3]. Consequently, much of the fundamental research has been performed towards understanding the mechanism behind the modification of the solidification structure by electric current [4-7]. However, the exact modification mechanism is not fully understood and it is still controversial.

To date, the main reasonable hypotheses proposed to explain the modification of the solidification structure are the increase in the solid-liquid interfacial tension [8], the reduction in the nucleation activation energy [9], the suppression of grain growth due to Joule heating [10], current crowding due to the differing electrical conductivities of solid and liquid [11], electromigration [12], and melt flow caused by Lorentz force [13], but all these taken together do not provide a consistent picture of the solidification structure evolution under electric current. This is mainly due to the lack of conclusive evidence provided by theoretical and experimental studies in support of the proposed hypotheses, especially in the solid-liquid interfacial tension and its related wettability of solid phase by liquid phase which play an important role in determining the kinetics of crystal nucleation and growth [14]. To better understand the intrinsic mechanism of applied electric current to the solidification structure of alloys, a knowledge of the correlation between current parameter and the solid-liquid interfacial tension during solidification process of alloys is required. However, very little experimental investigations have been performed on this issue.

To our knowledge, although as yet no measuring method is applicable for the solid-liquid interfacial tension when DC passes through the solid-liquid interface, recent studies on effect of an applied DC on the solid-liquid interfacial reactions in metal-metal system have given an implication for the determination of the solid-liquid interfacial tension and the wettability. Xu et al. [15] found that the application of DC could improve the wettability of molten $\mathrm{Bi}$ on $\mathrm{Cu}$ substrate using a DC-coupled sessile drop method, and current polarity 


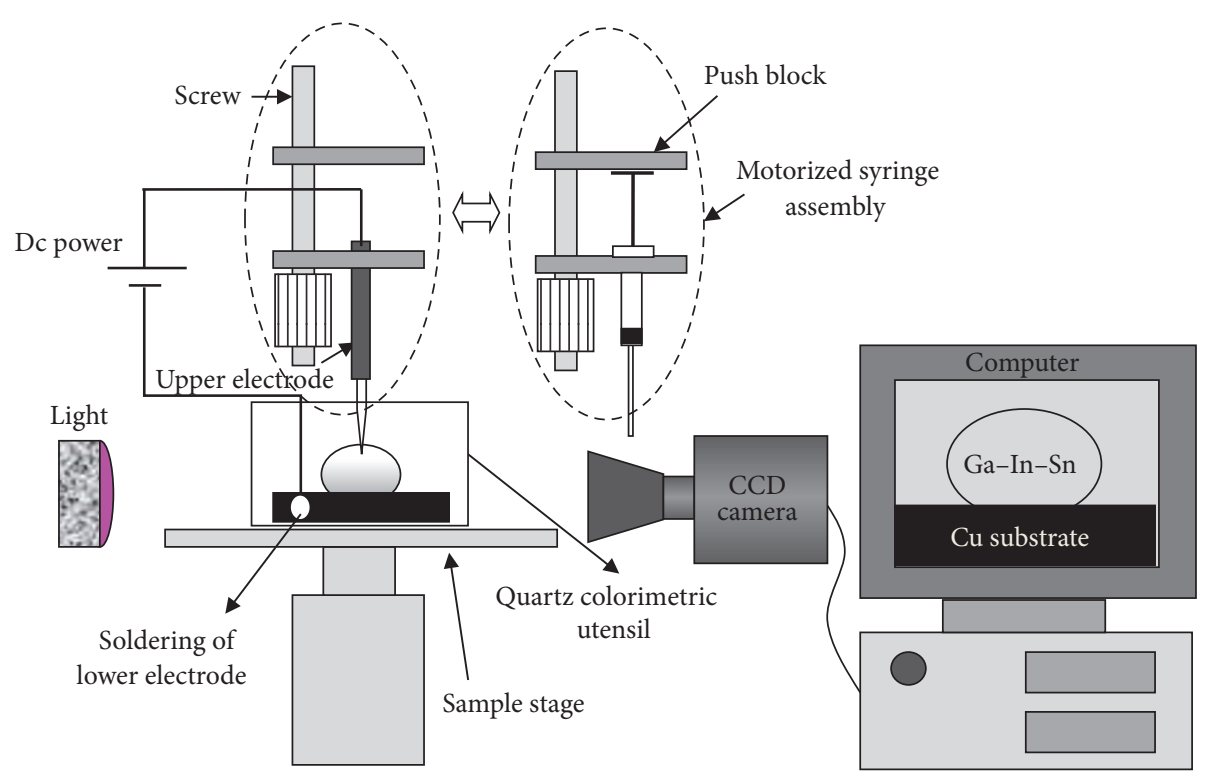

FIGURE 1: Schematic illustration of direct current-sessile drop apparatus.

did not give rise to significant difference on the wettability. Here, it should be pointed out that there are some drawbacks to their designed experiment. For example, the dissolution of the $\mathrm{Cu}$ electrode in liquid $\mathrm{Sn}$ at the setting temperature needs to be taken into account, and thus the wetting result is blemished. $\mathrm{Gu}$ et al. $[16,17]$ solved the above problem by selecting the graphite electrode to replace the $\mathrm{Cu}$ electrode. It was observed that similar results were obtained for the wettability of molten Sn on Cu substrate or Fe substrate. Moreover, the applied DC promoted the dissolution of the $\mathrm{Cu}$ substrate in molten Sn which was enhanced with increasing current intensity. It prevented the determination of solid-liquid interfacial tension from contact angles through Young's equation.

The aim of the present work is to obtain quantitative data on the wettability of $\mathrm{Cu}$ by molten alloy using a DCcoupled sessile drop method and to compare the solid-liquid interfacial tension under different current conditions. For the work, Ga-25In-13Sn ternary near-eutectic alloy is chosen as droplet at room temperature, because this alloy is liquid due to its low melting point. In this situation, the dissolution of the upper electrode in liquid alloy and the solid-liquid interfacial reaction can be neglected. This makes it possible to reveal the influence of DC on the solid-liquid interfacial tension and the wettability for the explanation of the solidification structure evolution under electric current. In addition, the potential mechanism of DC on the change of solid-liquid interfacial tension and wettability is also discussed in detail.

\section{Experimental Procedure}

The Ga-25In-13Sn (all percentages are wt- $\%$ unless otherwise stated) ternary near-eutectic alloy was prepared using highpurity metals of $\mathrm{Ga}$ (>99.999\%), In (>99.99\%), and $\mathrm{Sn}$ $(>99.99 \%)$ in a resistance furnace. After allowing time for melt homogenization, the alloy was taken out and aspirated into a microinjector. Pure $\mathrm{Cu}$ plates $(>99.9 \%)$ in a size of
$15 \times 15 \times 4 \mathrm{~mm}^{3}$ were used as substrates. They were mechanically polished to a mirror surface with roughness of a few nanometers using diamond pastes and then ultrasonically cleaned in acetone. The measurements of contact angle and surface tension under DC were performed using a DCcoupled sessile drop apparatus, as schematically shown in Figure 1. As can be seen, it was comprised of two main devices: a high speed (frame rate $=200 \mathrm{~Hz}$ ) contact angle instrument (Powereach, China) and the electric transmission mission device consisting of a DC power and a Cu wire of $\Phi 1.8 \mathrm{~mm}$ with a sharp-pointed head used as the upper electrode and $\mathrm{Cu}$ substrate, on the side of which the $\mathrm{Cu}$ wire was held, used as the bottom electrode. $\mathrm{Cu}$ substrate was put at the bottom of quartz colorimetric utensil and horizontally placed on the sample stage. Subsequently, liquid drop of Ga-In-Sn alloy with a volume of $40 \mu \mathrm{l}$ was injected on the $\mathrm{Cu}$ substrate surface with microinjector. After that the upper electrode, which replaced the position of microinjector, was fixed to the motorized syringe assembly. The sample stage can move in both horizontal and vertical direction to achieve a sufficient contact of the upper electrode with liquid drop.

In DC-coupled sessile drop experiments, the sharppointed upper electrode was initially moved down to be inserted into the liquid drop. This moment was defined as the starting time of the wettability (i.e., $t=0 \mathrm{~s}$ ), and then let the liquid drop stand for 5 minutes without DC. After that, DC of $1 \mathrm{~A}$ with a predetermined polarity was continuously applied for 5 minutes at room temperature, and then the $2 \mathrm{D}$ projection of $3 \mathrm{D}$ drop was recorded by a high speed framing camera. Repeating the above operations, each time current intensity added $1 \mathrm{~A}$ until current intensity increased to $11 \mathrm{~A}$. Due to the existence of relaxation time for the steady-state contact angle, the wetting experiment without DC was performed at room temperature and image was taken by the camera every 5 minutes, one which lasted until 65 minutes. Contact angles were calculated from the 


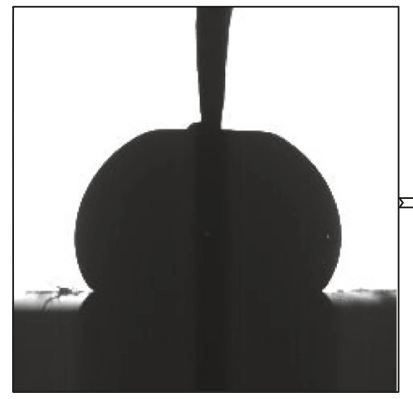

Original image

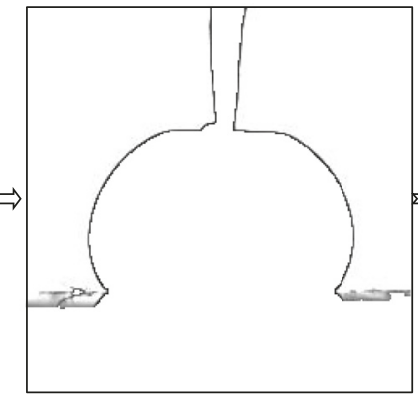

Extraction of the contours

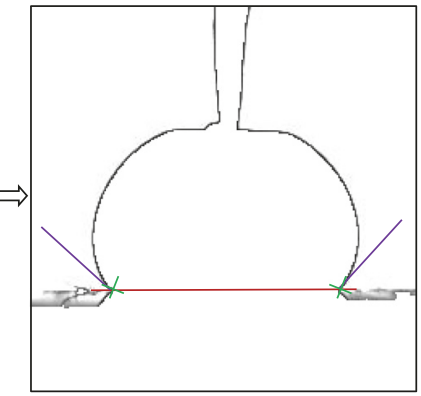

Baseline, intersections and tangent

FIGURE 2: Measurement of contact angle using image-processing techniques.

captured images using drop shape analysis. First the drop profile and the surface of $\mathrm{Cu}$ substrate were extracted using image-processing techniques, and then the intersection of the baseline with the tangent was obtained. The different steps in the processing technique are presented in Figure 2.

\section{Results and Discussion}

In order to conveniently describe current polarity, the direction of DC is defined as a positive one with the $\mathrm{Cu}$ substrate connected to the cathode, while the corresponding negative $\mathrm{DC}$ is one with the $\mathrm{Cu}$ substrate connected to the anode. Typical liquid drop shapes of $\mathrm{Ga}-\mathrm{In}-\mathrm{Sn}$ alloy on $\mathrm{Cu}$ substrate under different conditions are illustrated in Figure 3. Figures 3(a)-3(c) represent the metal drop spreading on $\mathrm{Cu}$ substrate at different time without DC; the corresponding drop contours are overlapped in Figure 3(d). It can be seen that wetting degree of $\mathrm{Cu}$ substrate by $\mathrm{Ga}-\mathrm{In}-\mathrm{Sn}$ alloy drop is poor and does not vary with time in the absence of DC. It suggests that metal drop spreading on $\mathrm{Cu}$ substrate forms the quasi-equilibrium shape within 5 minutes. Since the spreading rate was accelerated by DC according to $[15,16]$, the time required to form the approximate equilibrium shape during application of DC should be less than 5 minutes. In the present experiment, the duration of DC is 5 minutes in order to ensure sufficient spreading of $\mathrm{Ga}-\mathrm{In}-\mathrm{Sn}$ alloy on $\mathrm{Cu}$ substrate. Effect of positive DC on the wetting behavior of liquid drop of $\mathrm{Ga}$-In-Sn alloy on $\mathrm{Cu}$ substrate is depicted in Figures 3(e)-3(h), while the corresponding results under the condition of a negative DC are shown in Figures 3(i)-3(1). The wettability is still poor when DC is applied regardless of current polarity. However, the application of DC of +6 A deforms the drop shape compared with that without DC, and there is no increase in the deformation of drop when current intensity increases to $+11 \mathrm{~A}$, as shown in Figure 3(h). Similar deformation of drop is obtained upon DC reversal (Figure 3(1)).

Since wettability is usually characterized by contact angle of liquid on solid surface in three-phase equilibrium, contact angle measurements are necessary to profoundly understand effect of DC on the wettability of Cu substrate by $\mathrm{Ga}-\mathrm{In}-\mathrm{Sn}$ alloy melt. The plot of contact angle as a function of current intensity at room temperature is shown in Figure 4. As a result, DC reduces the contact angle regardless of current polarity. The decrease of contact angle with increasing current intensity is approximately linear when the applied DC is not more than $6 \mathrm{~A}$ in current intensity, and there is a critical value for current intensity, above which contact angle remains almost constant. Moreover, current polarity seems to have no significant effect on contact angle.

According to Young's equation (1), the contact angle of liquid drop on solid surface is determined by the force balance between the interfacial tensions at the solid-liquidgas interface:

$$
\gamma_{\mathrm{lg}} \cos \theta=\gamma_{\mathrm{sg}}-\gamma_{\mathrm{sl}}
$$

where $\theta$ is the Young contact angle, $\gamma_{\mathrm{lg}}$ is the liquid-gas surface tension, $\gamma_{\mathrm{sg}}$ is the solid-gas surface tension, and $\gamma_{\mathrm{sl}}$ is the solid-liquid interfacial tension. It should be pointed out that (1) is only applicable to thermodynamically meaningful contact angles [18]. In that case, it suggests that the observed decrease in contact angles is a manifestation of the effect of DC on interfacial tensions. It is very probable that the solidgas surface tension is unaffected by DC due to the lack of mobility of atom of the solid. Therefore, it is believed that the observed decrease in contact angles is a consequence of a change in liquid-gas and solid-liquid interfacial tensions.

Taking the solid-gas surface tension as a constant, effect of DC on contact angle can be translated into the corresponding effects in terms of the liquid-gas and solid-liquid interfacial tensions using the equation of state [19]:

$$
\begin{aligned}
& \gamma_{\mathrm{lg}}=\frac{2 \gamma_{\mathrm{Sg}}}{\left(\sqrt{1+\sin ^{2} \theta}+\cos \theta\right)}, \\
& \gamma_{\mathrm{sl}}=\frac{\gamma_{\mathrm{Sg}}\left(\sqrt{1+\sin ^{2} \theta}-\cos \theta\right)}{\left(\sqrt{1+\sin ^{2} \theta}+\cos \theta\right)} .
\end{aligned}
$$

Equation (2) describes the dependence of liquid-gas and solid-liquid interfacial tensions on contact angle, respectively. They can be employed to qualitatively estimate the change of liquid-gas and solid-liquid interfacial tensions under DC compared with those without DC. Figure 5 shows variation of the calculated solid-liquid and liquid-gas interfacial tensions ratio with current intensity and polarity. Obviously, with increasing current density, the ratio of calculated solid-liquid interfacial tension $\left(\gamma_{\mathrm{sl}}^{e}\right)$ under DC to that $\left(\gamma_{\mathrm{sl} 0}\right)$ in the absence of DC decreases at first and tends to a constant when 


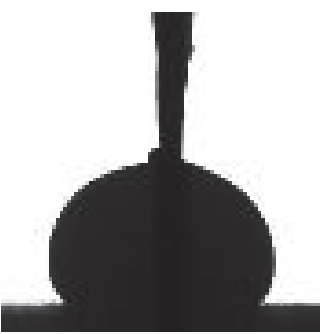

(a)

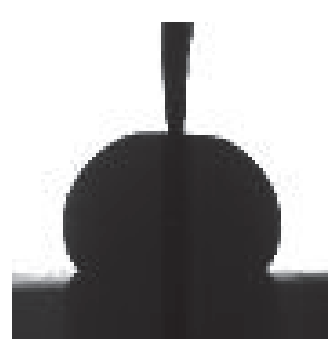

(e)

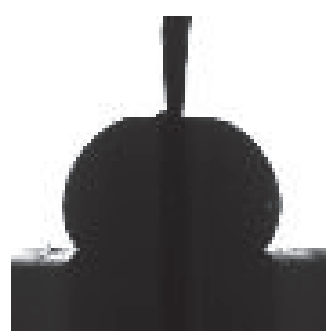

(i)

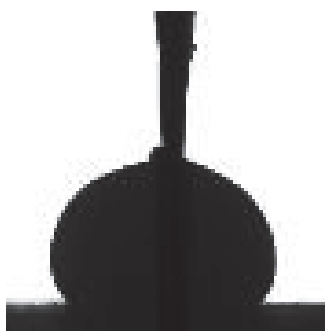

(b)

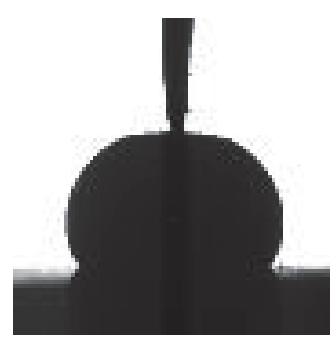

(f)

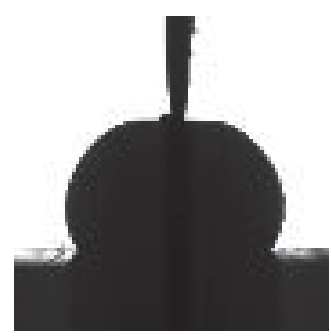

(j)

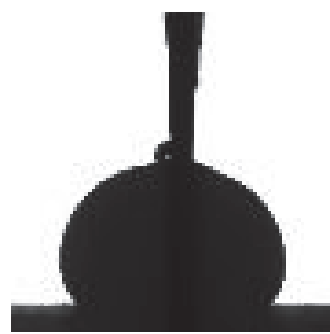

(c)

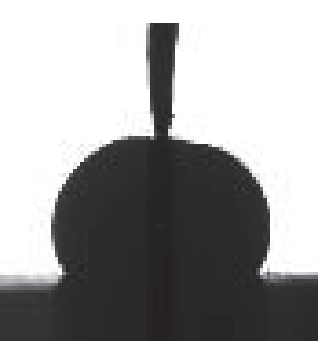

$-I=0 \mathrm{~A}$

$--I=+6 \mathrm{~A}$

$--I=+11 \mathrm{~A}$

(g)

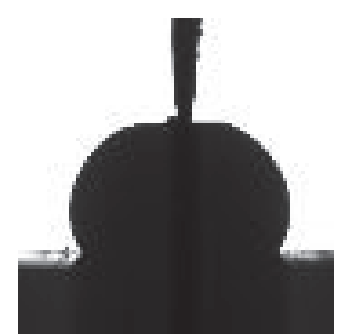

$\begin{aligned}-I & =0 \mathrm{~A} \\ ---I & =-6 \mathrm{~A} \\ ---I & =-11 \mathrm{~A}\end{aligned}$

(k)

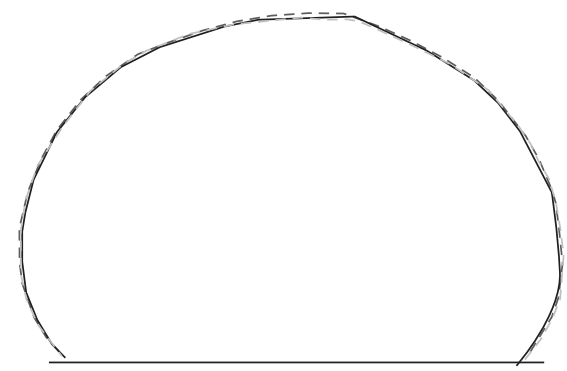

(d)



(h)

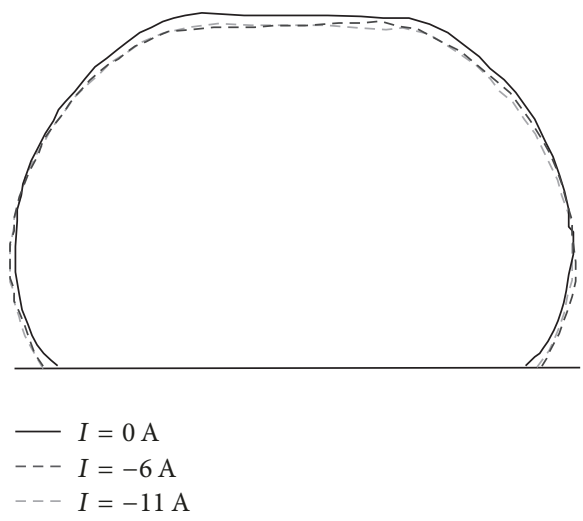

(l)

Figure 3: Typical liquid Ga-In-Sn drop shapes on Cu substrate under different conditions. Without DC after different standing time of (a) $5 \mathrm{~min}$, (b) $35 \mathrm{~min}$, and (c) $65 \mathrm{~min}$, the three aforementioned drop shapes are amplified and overlapped in Figure (d). With positive DC (e) $0 \mathrm{~A}$, (f) $6 \mathrm{~A}$, and (g) $11 \mathrm{~A}$, the three aforementioned drop shapes are amplified and overlapped in Figure (h). With negative DC (i) $0 \mathrm{~A}$, (j) $6 \mathrm{~A}$, and (k) $11 \mathrm{~A}$, the three aforementioned drop shapes are amplified and overlapped in Figure (l).

current intensity exceeds $6 \mathrm{~A}$ regardless of current polarity (Figure 5(a)). Similar tendency is observed for the ratio of calculated liquid-gas interfacial tension $\left(\gamma_{\mathrm{lg}}^{e}\right)$ under DC to that $\left(\gamma_{\mathrm{lg} 0}\right)$ in the absence of DC. It indicates that the observed change in contact angle is a consequence of the decrease in the liquid-gas and solid-liquid interfacial tensions.

Since the measurement of contact angle is performed using a DC-coupled sessile drop method at room temperature, the mass transfer and interfacial action at the triplephase region are negligible. In this case, Young's equation and its extended formulas are applicable to determinate liquid-gas and solid-liquid interfacial tensions from contact angle in the present study. As indicated above, the observed decrease in contact angle results from the decrease of liquid interfacial tensions. The potential mechanism causing the above change in liquid interfacial tensions refers to a series of current effects such as Joule heating, electromigration, electric potential energy, and convection caused by Lorentz force. Therefore, the contributions of these effects to liquid interfacial tensions should be seriously considered.

When electric current passes through a conductor, Gibbs free energy increases by adding an extra term $\left(\delta G_{e}\right)$ at the same temperature $[20,21]$. It is speculated that solute atoms in alloys will be excited to a higher energy state under DC and affect the solute diffusion process. An explanation to the influence of DC on the diffusion activation energy $(Q)$ of 


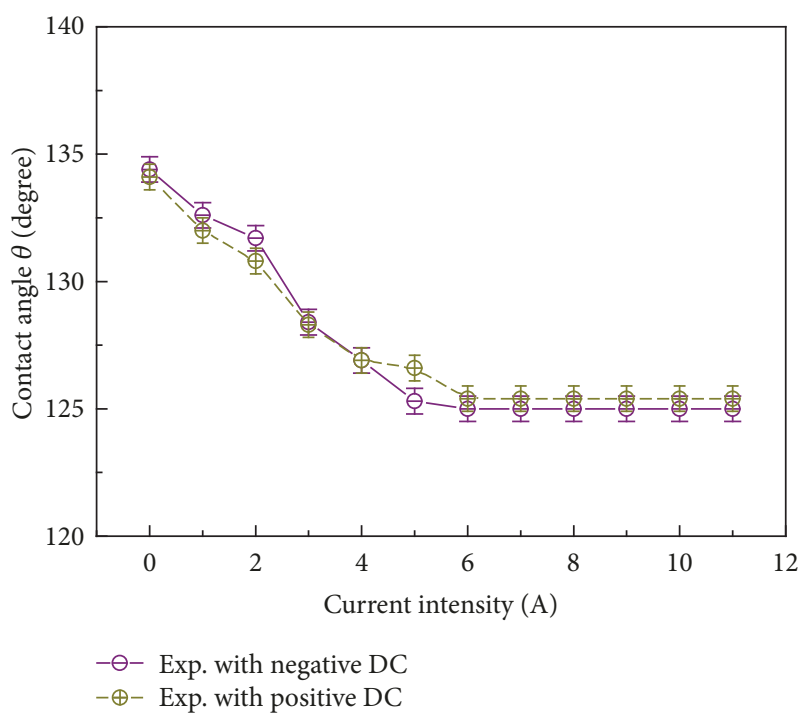

FIGURE 4: Contact angle versus current intensity and polarity.

solute is schematically illustrated in Figure 6(a). Generally, the diffusion activation energy, sometimes called the diffusion barrier, is defined as the minimum energy required to start atom migration. Position 1 and position 2 represent the initial location and target location of atom migration, respectively. In the absence of DC, the diffusion barrier $(\Delta G)$ is given by $\Delta G=G_{2}-G_{1}$. The diffusion barrier $\left(\Delta G_{e}\right)$ decreases under DC due to the contribution of an extra term $\left(\delta G_{e}\right)$. It suggests that DC reduces the diffusion activation energy of solute and then increases its diffusion coefficient $(D)$ at the same temperature in terms of the relationship between $D, Q$, and absolute temperature ( $T$ ) [22]. Similarly, Zhao and coworkers have found that the calculated diffusion coefficient for solid $\mathrm{Ni}$ in liquid $\mathrm{Al}$ increases with increasing current density under DC and approaches a relatively stable value at a certain critical current density, regardless of current polarity [23]. It is concluded that DC plays an important role in promoting the diffusion process of metal atoms.

Additionally, it should be noted that the physical parameters of liquid alloys, liquid-gas interfacial tension $\left(\gamma_{\text {lg0 }}\right)$, viscosity $(\eta)$, and solute diffusion coefficient $(D)$ are intensively correlative. Therefore, the relationship between $\gamma_{\mathrm{lg} 0}, \eta$, and $D$ can be given with some empirical models [22]:

$$
\begin{aligned}
\eta & =\frac{16}{15} \sqrt{\frac{M}{k T}} \cdot \gamma_{\mathrm{lg} 0}, \\
D & =\frac{k T}{6 \pi r \eta},
\end{aligned}
$$

where $M$ is the absolute atomic mass, $k$ is the Boltzmann constant, and $r$ is the characteristic radius of solute atom. According to (3), the liquid-gas interfacial tension is inversely proportional to the solute diffusion coefficient, implying that the DC-induced promotion of solute diffusion coefficient obviously decreases the liquid-gas interfacial tension of liquid Ga-In-Sn alloy regardless of current polarity, which is in agreement with the calculated results. As indicated above, the changing trends for solid-liquid and liquid-gas interfacial tensions are similar regarding current intensity dependence. Thus, it is likely that the reduction of diffusion activation energy is the main factor for the reduction in contact angle and solid-liquid interfacial tension under DC.

Since previous studies have proved that Joule heating, electromigration, and convection caused by Lorentz force play significant roles in determining the dissolutive wetting process of molten metal on solid substrate at higher temperature $[15,16]$, it is worth studying how to affect the wettability of $\mathrm{Cu}$ by liquid $\mathrm{Ga}-\mathrm{In}-\mathrm{Sn}$ alloy and its related solid-liquid interfacial tension at room temperature by the above-mentioned current effects. Here, current density as an important parameter in assessing current effects is essential to be determined and its average values are estimated as $10-110 \mathrm{Acm}^{-2}$ in our experiment. In prior work it was observed that effect of Joule heating effect on temperature was negligible for $\mathrm{Sn}-\mathrm{Bi}$ alloy under DC of $50 \mathrm{Acm}^{-2}$. The change of temperature $(\Delta T)$ under DC is proportional to current density squared $\left(j^{2}\right)$ and electrical resistivity of materials $\left(\rho_{e}\right.$, approximately $7.0 \times 10^{-7} \Omega \cdot \mathrm{m}$ for $\mathrm{Sn}-\mathrm{Bi}$ alloy at $500 \mathrm{~K}$ [24] and $2.9 \times 10^{-7} \Omega \cdot \mathrm{m}$ for Ga-In-Sn alloy at room temperature [25]) [26]. As a consequence, the obvious change of temperature occurs only when current density exceeds about $120 \mathrm{Acm}^{-2}$ in this study. Thus, effect of Joule heating in liquid drop on the wettability should be negligible with the present DC range of 1-11 A. Generally, current densities of the order of $10^{2} \mathrm{Acm}^{-2}$ are required to produce a substantial electromigration, which enhances with increasing current density [12]. Composition fluctuation at the solid-liquid interface as the direct expression of electromigration [4] will affect the solid-liquid interfacial tension and depends on current polarity. Therefore, the difference of the solid-liquid interfacial tension occurs upon DC reversal and increases with increasing current density, which is inconsistent with our experimental results. It suggests that the contribution of electromigration is not expected to be significant to decrease the solid-liquid interfacial tension. As has been reported before, Lorentz force caused by the interaction between the applied DC and its own induced magnetic field gives rise to significant convection in liquid phase [23]. In our case, flow field in the drop, which is similar to that reported in [27], provides a driving force for composition homogenization and weakens composition fluctuation at the solid-liquid interface due to electromigration. According to drop shape, the liquid near the upper electrode has a higher current density, which is estimated as $40-440 \mathrm{Acm}^{-2}$. It implies that temperature gradient as a result of differences in Joule heating associated with differences in cross-sectional area of drop should be considered when current intensity of the applied DC exceeds $3 \mathrm{~A}$, which can lead to Marangoni convection in drop, as schematically shown in Figure 6(b). Note that there is no significant difference between the direction of Marangoni convection and that of convection induced by Lorentz force. Marangoni convection also plays a role in restraining composition fluctuation at the solid-liquid interface. Suppression of electromigration by convection explains the fact that there is no distinction in the wettability upon DC reversal. 


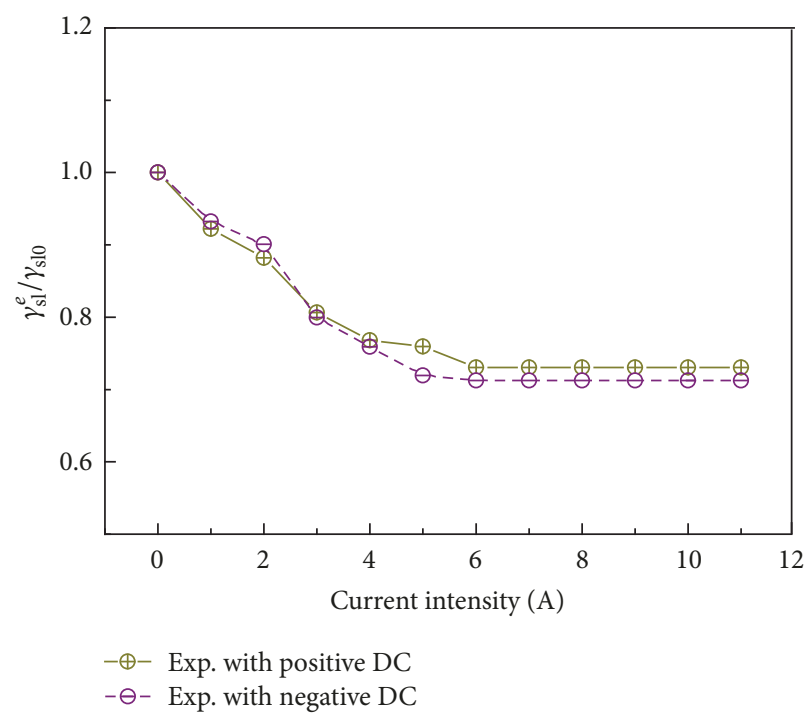

(a)

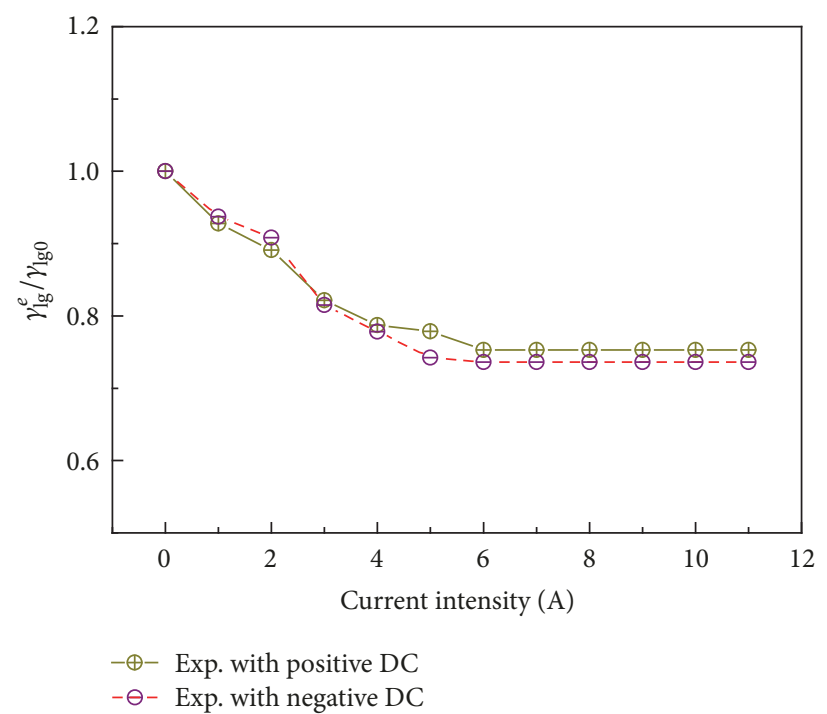

(b)

FIGURE 5: (a) Variation of solid-liquid interfacial tension ratio $\left(\gamma_{\mathrm{sl}}^{e} / \gamma_{\mathrm{sl} 0}\right)$ with current intensity and polarity. (b) Variation of liquid-gas surface tension $\left(\gamma_{\mathrm{lg}}^{e} / \gamma_{\mathrm{lg} 0}\right)$ with current intensity and polarity.

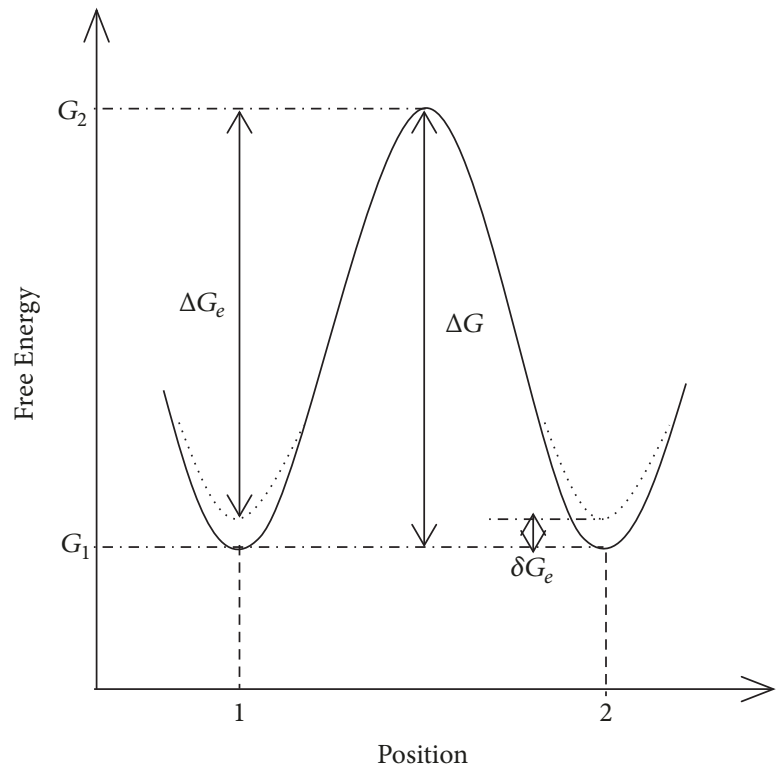

(a)

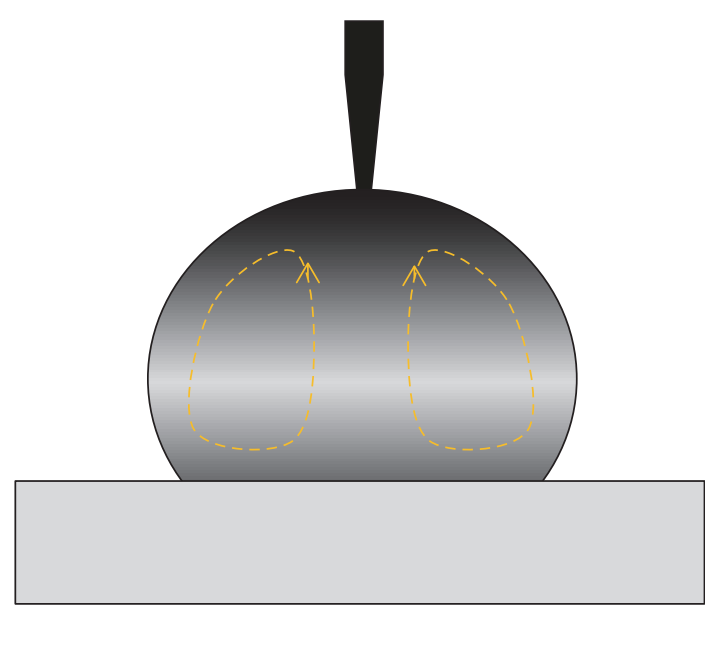

(b)

Figure 6: (a) Schematic explanation for the effect of DC on the diffusion activation energy. (b) Schematic of Marangoni convection in the $\mathrm{Ga}-\mathrm{In}-\mathrm{Sn} / \mathrm{Cu}$ system under DC.

\section{Conclusions}

We have investigated the wetting behavior of $\mathrm{Ga}-\mathrm{In}-\mathrm{Sn}$ alloy melt on $\mathrm{Cu}$ substrate and solid-liquid interfacial tension under DC at room temperature. The application of DC has a pronounced effect on the wettability. There is a critical value for current intensity, below which the steady-state contact angle decreases with increasing current intensity and above which contact angle remains almost constant. In addition, the direction of DC does not play a role in the observed effects under the same current intensity. Using the equation of state that describes the dependence of solid-liquid interfacial tension on contact angle, the change of solid-liquid interfacial tension under DC can be qualitatively determined. The application of DC leads to significant reduction of solidliquid interfacial tension. It is speculated that the reduction of contact angle and its corresponding solid-liquid interfacial tension is mainly attributed to the promotion of solute diffusion coefficient under DC. Moreover, Joule heating, Marangoni convection induced by nonuniform distribution 
of temperature filed, electromigration, and convection caused by Lorentz force do not have a noticeable effect.

\section{Conflicts of Interest}

The authors declare that there are no conflicts of interest regarding the publication of this paper.

\section{Acknowledgments}

This work is supported by the National Natural Science Foundation of China (Grant nos. 51704242 and 51701160), the Young Scholars Program of Shandong University (Weihai), and the NPU Foundation for Fundamental Research in China (JC201272).

\section{References}

[1] T. Wang, J. Xu, T. Xiao et al., "Evolution of dendrite morphology of a binary alloy under an applied electric current: An in situ observation," Physical Review E: Statistical, Nonlinear, and Soft Matter Physics, vol. 81, no. 4, Article ID 042601, 2010.

[2] S. R. Coriell, G. B. McFadden, A. A. Wheeler, and D. T. J. Hurle, "The effect of an electric field on the morphological stability of the crystal-melt interface of a binary alloy. II. Joule heating and thermoelectric effects," Journal of Crystal Growth, vol. 94, no. 2, pp. 334-346, 1989.

[3] M. Nakada, Y. Shiohara, and M. C. Flemings, "Modification of Solidification Structures by Pulse Electric Discharging," ISIJ International, vol. 30, no. 1, pp. 27-33, 1990.

[4] L. Zhang, N. Li, R. Zhang et al., "Effect of Direct Current on Microstructure Evolution of Directionally Solidified Sn-70 wt pct Bi Alloy at Different Pulling Rates," Metallurgical and Materials Transactions A: Physical Metallurgy and Materials Science, vol. 46, no. 9, pp. 4174-4182, 2015.

[5] X. Liao, Q. Zhai, J. Luo, W. Chen, and Y. Gong, "Refining mechanism of the electric current pulse on the solidification structure of pure aluminum," Acta Materialia, vol. 55, no. 9, pp. 3103-3109, 2007.

[6] D. Räbiger, Y. Zhang, V. Galindo, S. Franke, B. Willers, and S. Eckert, "The relevance of melt convection to grain refinement in Al-Si alloys solidified under the impact of electric currents," Acta Materialia, vol. 79, pp. 327-338, 2014.

[7] H. Conrad, "Influence of an electric or magnetic field on the liquid-solid transformation in materials and on the microstructure of the solid," Materials Science and Engineering: A Structural Materials: Properties, Microstructure and Processing, vol. 287, no. 2, pp. 205-212, 2000.

[8] X. F. Zhang, W. J. Lu, and R. S. Qin, "Removal of MnS inclusions in molten steel using electropulsing," Scripta Materialia, vol. 69, no. 6, pp. 453-456, 2013.

[9] L. Jianming, L. Shengli, L. Jin, and L. Hantong, "Modification of solidification structure by pulse electric discharging," Scripta Materialia, vol. 31, no. 12, pp. 1691-1694, 1994.

[10] J. Zhu, T. Wang, F. Cao, W. Huang, H. Fu, and Z. Chen, "Real time observation of equiaxed growth of $\mathrm{Sn}-\mathrm{Pb}$ alloy under an applied direct current by synchrotron microradiography," Materials Letters, vol. 89, pp. 137-139, 2012.

[11] C. Song, Y. Guo, Y. Zhang et al., "Effect of currents on the microstructure of directionally solidified Al4.5 wt $\% \mathrm{Cu}$ alloy," Journal of Crystal Growth, vol. 324, no. 1, pp. 235-242, 2011.
[12] L. Zhang, N. Li, H. Xing et al., "Microstructure evolution of directionally solidified Sn-Bi alloy under different mediumdensity direct current," Journal of Crystal Growth, vol. 430, pp. 80-86, 2015.

[13] L. Zhang, H. Liu, N. Li et al., "The relevance of forced melt flow to grain refinement in pure aluminum under a low-frequency alternating current pulse," Journal of Materials Research, vol. 31, no. 3, pp. 396-404, 2016.

[14] K. Mondal and B. S. Murty, "On the prediction of solid-liquid interfacial energy of glass forming liquids from homogeneous nucleation theory," Materials Science and Engineering: A Structural Materials: Properties, Microstructure and Processing, vol. 454-455, pp. 654-661, 2007.

[15] Q.-G. Xu, X.-B. Liu, and H.-F. Zhang, "Effect of direct electric current on wetting behavior of molten $\mathrm{Bi}$ on $\mathrm{Cu}$ substrate," Transactions of Nonferrous Metals Society of China, vol. 20, no. 8, pp. 1452-1457, 2010.

[16] Y. Gu, P. Shen, N.-N. Yang, and K.-Z. Cao, "Effects of direct current on the wetting behavior and interfacial morphology between molten $\mathrm{Sn}$ and $\mathrm{Cu}$ substrate," Journal of Alloys and Compounds, vol. 586, pp. 80-86, 2014.

[17] P. Shen, Y. Gu, N.-N. Yang, R.-P. Zheng, and L.-H. Ren, "Influences of electric current on the wettability and interfacial microstructure in Sn/Fe system," Applied Surface Science, vol. 328, pp. 380-386, 2015.

[18] D. Y. Kwok and A. W. Neumann, "Contact angle measurement and contact angle interpretation," Advances in Colloid and Interface Science, vol. 81, no. 3, pp. 167-249, 1999.

[19] D. Y. Zhu, P. Q. Pin, X. B. Luo et al., "Novel characterization of wetting properties and the calculation of liquid-solid interface tension (I)," Science Technology and Engineering, vol. 7, no. 13, pp. 3057-3062, 2007.

[20] R. S. Qin, E. I. Samuel, and A. Bhowmik, "Electropulse-induced cementite nanoparticle formation in deformed pearlitic steels," Journal of Materials Science, vol. 46, no. 9, pp. 2838-2842, 2011.

[21] X. L. Wang, J. D. Guo, Y. M. Wang, X. Y. Wu, and B. Q. Wang, "Segregation of lead in $\mathrm{Cu}-\mathrm{Zn}$ alloy under electric current pulses," Applied Physics Letters, vol. 89, no. 6, Article ID 061910, 2006.

[22] I. Egry, "On the relation between surface tension and viscosity for liquid metals," Scripta Materialia, vol. 28, no. 10, pp. 12731276, 1993.

[23] J. F. Zhao, C. Unuvar, U. Anselmi-Tamburini, and Z. A. Munir, "Kinetics of current-enhanced dissolution of nickel in liquid aluminum," Acta Materialia, vol. 55, no. 16, pp. 5592-5600, 2007.

[24] X.-F. Li, F.-Q. Zu, H.-F. Ding, J. Yu, L.-J. Liu, and Y. Xi, "Hightemperature liquid-liquid structure transition in liquid $\mathrm{Sn}-\mathrm{Bi}$ alloys: Experimental evidence by electrical resistivity method," Physics Letters A, vol. 354, no. 4, pp. 325-329, 2006.

[25] Y. Plevachuk, V. Sklyarchuk, S. Eckert, G. Gerbeth, and R. Novakovic, "Thermophysical properties of the liquid Ga-In-Sn eutectic alloy," Journal of Chemical \& Engineering Data, vol. 59, no. 3, pp. 757-763, 2014.

[26] L. M. Zhang, N. Li, R. Zhang, H. Xing, K. Song, and J. Y. Wang, "Effect of medium-density direct current on dendrites of directionally solidified $\mathrm{Pb}-50 \mathrm{Sn}$ alloy," Materials Science and Technology (United Kingdom), vol. 32, no. 18, pp. 1877-1885, 2016.

[27] P. A. Nikrityuk, K. Eckert, R. Grundmann, and Y. S. Yang, "An impact of a low voltage steady electrical current on the solidification of a binary metal alloy: A numerical study," Steel Research International, vol. 78, no. 5, pp. 402-408, 2007. 

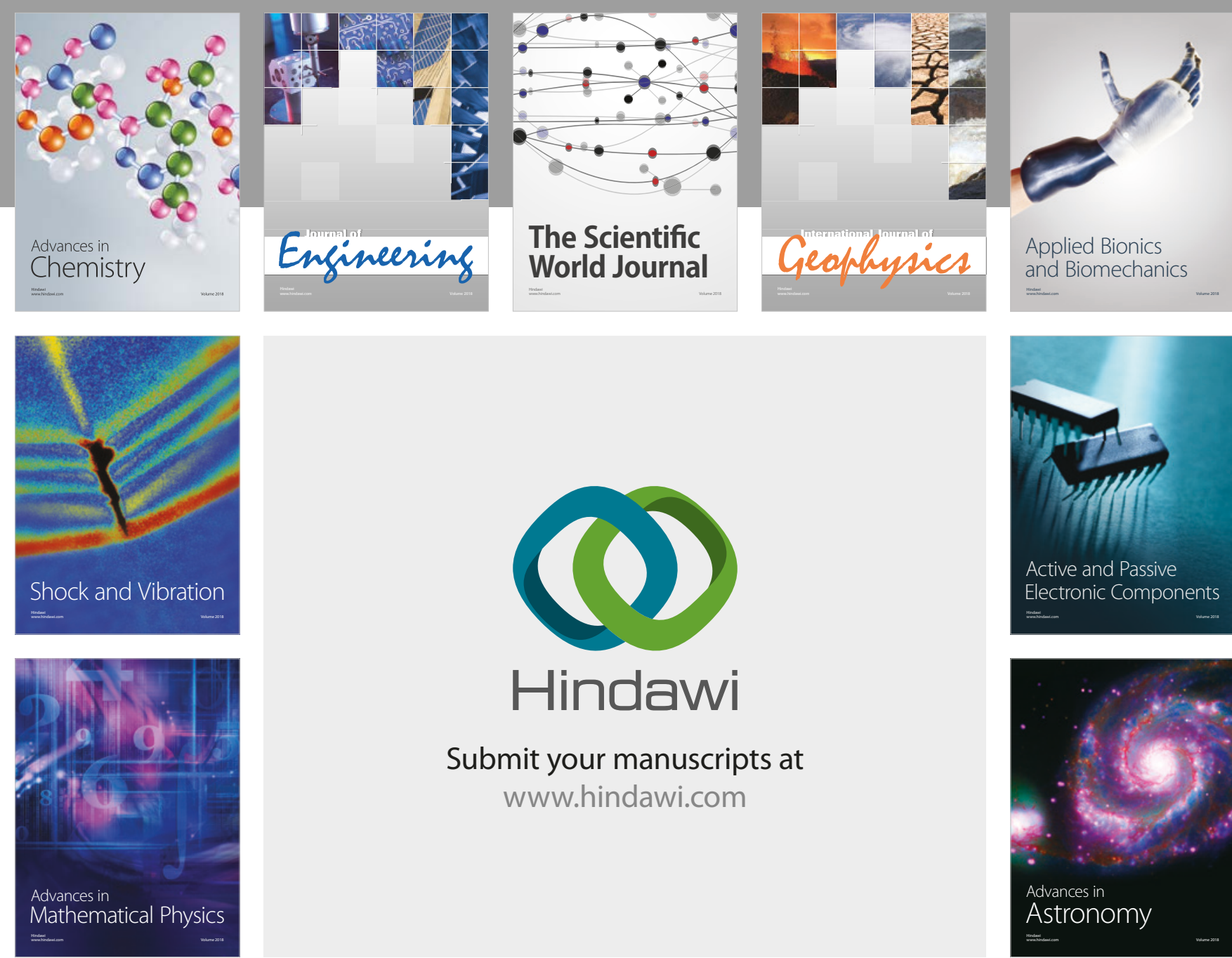

Submit your manuscripts at

www.hindawi.com

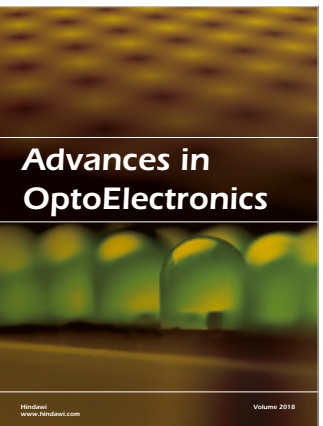

\section{Rotcting Machinery}
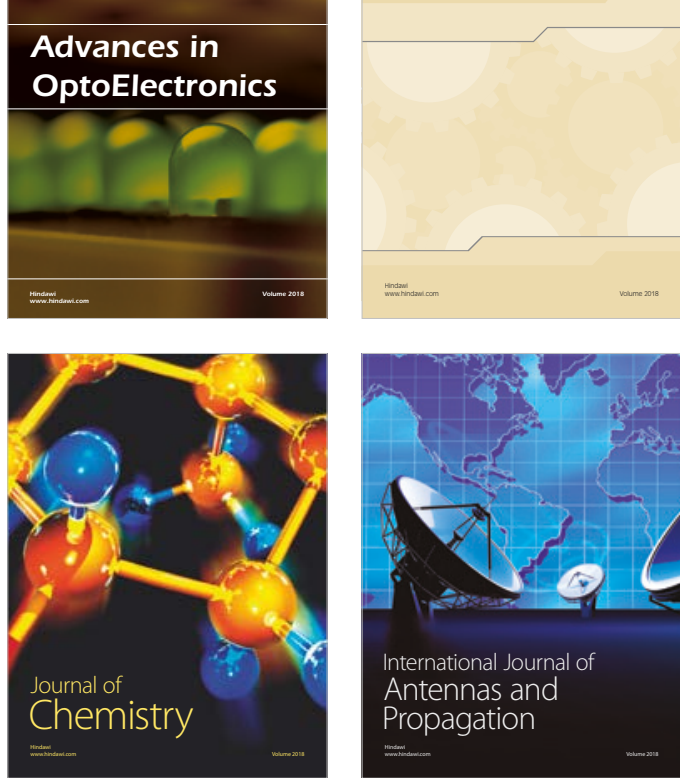



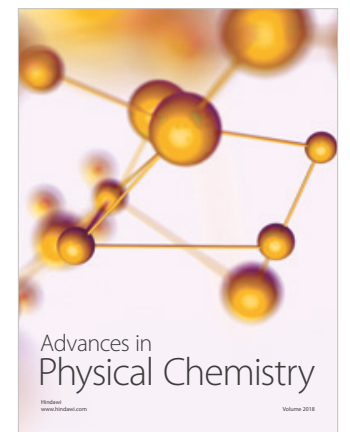

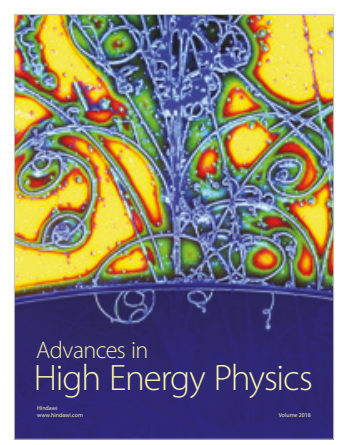

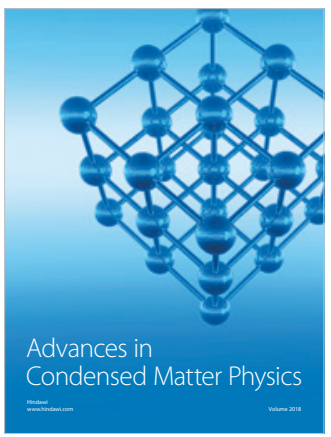

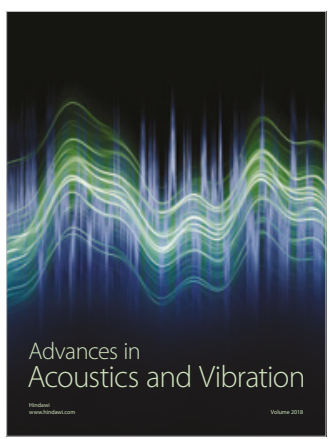

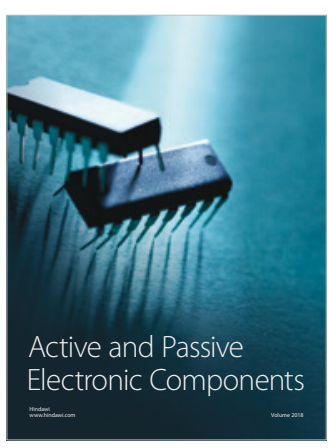
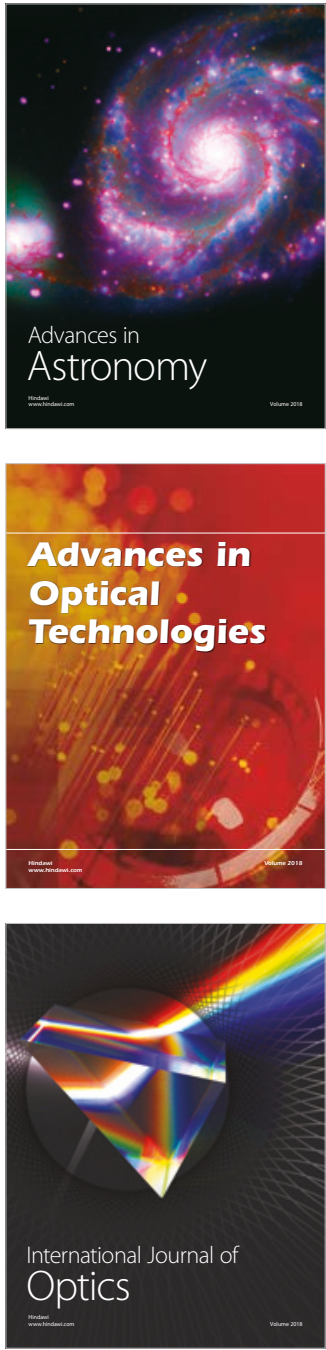